\title{
Egress Efficacy of Persons with Multiple Sclerosis During Simulated Evacuations
}

\author{
Richard M. Kesler*, Alexandra E. Kliegar, and Gavin P. Horn, Fire Service \\ Institute, University of Illinois, Urbana-Champaign, MC-675, 11 Gerty \\ Drive, Champaign, IL 61820, USA
}

Alexandra E. Klieger, Department of Fire Protection Engineering, University of Maryland, College Park, MD, USA

Morgan K. Boes and Elizabeth T. Hsiao-Wecksler, Department of

Bioengineering, University of Illinois, Urbana, IL, USA

Elizabeth T. Hsiao-Wecksler, Department of Mechanical Science and Engineering, University of Illinois, Urbana, IL, USA

Rachel E. Klaren, Department of Kinesiology and Community Health, University of Illinois, Urbana, IL, USA

Yvonne Learmonth, School of Psychology and Exercise Science, Murdoch University, Murdoch, WA, Australia

Robert W. Motl, Department of Physical Therapy, University of Alabama Birmingham, Birmingham, AL, USA

Received: 3 January 2017/Accepted: 4 July 2017

\begin{abstract}
Expedited evacuation of commercial and residential structures in the event of an emergency may be more difficult for persons with physical movement disorders. There is a need to better characterize the impact of such disorders and provide movement data to improve evacuee and responder safety. We undertook a pilot, feasibility study that investigated the ability of persons with multiple sclerosis (MS) and controls without MS to walk along a $48 \mathrm{~m}$ long path that included five different door configurations with various opening hardware and closure mechanisms, both before and after a six-minute walk, simulating a long evacuation path. Persons with MS took longer to complete the evacuation circuit (102 vs. $31 \mathrm{~s}$ ) and to pass through each door (average 4.8 vs. $1.4 \mathrm{~s}$ ) compared to controls. During the six-minute walk, persons with MS had decreased walking speed $(0.7$ vs. $1.9 \mathrm{~m} / \mathrm{s})$. The MS population demonstrated more conservative gait biomechanics throughout the simulation, i.e., wider, shorter and slower steps. Timing and biomechanical differences between populations and the potential fatigue induced through an extended evacuation can be used to improve understanding of movement in populations with disabilities, and incorporate these data into estimation of flow rates during evacuation.
\end{abstract}

Keywords: Evacuation, Multiple sclerosis, Movement disorders, Gait

\footnotetext{
* Correspondence should be addressed to: Richard M. Kesler, E-mail: rkesler2@illinois.edu
} 


\section{Introduction}

There are more than 9.5 million individuals in the United States with movement disorders (nearly four percent of the adult population) with that number projected to grow to 20 million (approximately five percent of the projected adult population) by 2050 with improvements in healthcare and an aging population [1]. Movement disorders can result from a large spectrum of origins including aging, obesity, Parkinson's disease, stroke, cerebral palsy, spinal cord injury, and traumatic brain injury among others. Many of these movement disorders are locomotion disabilities. Individuals with multiple sclerosis (MS) represent approximately 400,000 of these cases [2]. MS is an immune response targeted at the central nervous system leading to demyelination of the nerve cells. This may lead to degradation of the motor system and affect musculoskeletal movements. Further, MS can result in significant perceptions of fatigue, even when only a mild degree of disability is present [3]. Substantial research exists characterizing gait in the MS population [4-9] (e.g. those with MS walk slower with a lower cadence and exhibit wider, shorter steps than controls [4]) and these detriments may be particularly relevant during emergency conditions.

Boyce [10] emphasized that individuals with locomotion and other disabilities are increasingly present in society and active in public spaces. Increased Americans with Disabilities Act (ADA) compliance provides more access for those with movement disorders to these structures. Public spaces must be designed to accommodate the egress of these individuals based on their presence and building occupancy. At the same time, public, commercial and residential structures continue to increase in size and complexity. As such longer, more complex evacuation routes with multiple door passages may be encountered in order to protect egress pathways and compartmentalize structures. It is critical to understand how these long evacuation routes with potential to encounter multiple closed doors might impact the egress capability of those with locomotion disabilities who have increasing access.

As the prevalence of movement disorders increases in society, so does the need for research regarding the evacuation capabilities and characteristics of individuals with various movement disorders. Boyce and Shields pioneered studies regarding the characterization of building occupancies for fire safety of people with disabilities [10-14]. Gait speed for individuals with locomotive disabilities are consistently slower than their able-bodied counterparts, with decreasing speed as the severity of the impairment increases [11]. While studies have continued to examine movement of individuals in large and potentially complex structures such as high-rise buildings $[15,16]$, limited recent research has focused on evaluation of those with movement disorders $[17,18]$.

Recent work by the National Institute for Standards and Technology regarding evacuation for individuals with mobility impairments has emphasized the lack of existing data that can be used to estimate required safe egress time (RSET) for comparison with the available safe egress time (ASET) in fire scenarios common in today's structures. ASET and RSET are essential components of any egress 
model and their accuracy must be improved for performance-based building design [17]. These egress models often rely on available data regarding the movement capabilities, movement independence, and occupied space of the individuals within the environment. Further characterizing these parameters can result in an increased awareness of population needs and more accurate models for improved life safety [19].

This pilot, feasibility study is novel in that it is the first examination of gait in persons with multiple sclerosis during simulated emergencies and directly compares these experimental measurements to a control group. It was hypothesized that persons with MS would take significantly longer to complete a simulated 'evacuation circuit' consisting of walking through a course that included multiple door opening obstacles and that this completion time would be positively correlated with increasing levels of disability [based on the expanded disability status scale (EDSS)]. Furthermore, it was hypothesized that the MS population would exhibit wider, shorter steps than age-matched controls [4] and that an age-matched control group would complete the evacuation circuit slower and exhibit wider, shorter steps while travelling slower than a young control group. Lastly, the authors anticipated that, after performing a six-minute walk (simulating an extended duration evacuation), the completion time of a subsequent evacuation circuit would be slower.

\section{Methods}

\subsection{Sample Descriptions}

Fifteen adults with MS were recruited for the study with varying severities of disease progression as measured by the Expanded Disability Status Scale (EDSS) [20] ranging from 3.5 to 6.5 . These 15 adults provided a range of disability levels amongst those with MS who are ambulatory without assistance from others. Fifteen age-, weight- and height-matched controls (AMC) and 12 young adult controls (YC) were recruited to examine the effects of aging in addition to disease progression (Table 1). This sample size was selected as this was a feasibility, pilot study on egress in MS. The recruited sample size and resulting data provide adequate power to make initial conclusions and supply a baseline data set for power calculations in future studies. Importantly, the authors hope this data set will prompt further examination of egress in MS, particularly considering the novelty of this approach and area.

\section{Table 1}

Subject Descriptives (Mean \pm SD)

\begin{tabular}{lllll}
\hline Group & $\mathrm{N}$ & Age (years) & Weight $(\mathrm{kg})$ & Height $(\mathrm{cm})$ \\
\hline Multiple sclerosis (MS) & 15 & $54.3 \pm 5.3$ & $83.2 \pm 28.3$ & $170.2 \pm 8.8$ \\
Age matched controls (AMC) & 15 & $49.7 \pm 12.0$ & $81.6 \pm 19.4$ & $171.8 \pm 11.6$ \\
Young controls (YC) & 12 & $21.0 \pm 1.2$ & $70.4 \pm 5.8$ & $175.0 \pm 7.8$ \\
\hline
\end{tabular}




\subsection{Experimental Design and Instrumentation}

The experiment consisted of four phases: (1) familiarization, (2) initial evacuation circuit (EC), (3) a Six-Minute Walk (6 MW), and (4) post-walk EC. All subjects wore comfortable clothes and shoes throughout the study. The Institutional Review Board of the University of Illinois approved the protocol.

Phase 1 provided each subject with an opportunity to become familiarized with the EC arrangement, door operation, and instrumentation. The EC was demonstrated to the subject whilst the subject was seated in a wheelchair.

During Phase 2, subjects completed an initial evacuation circuit in a rested state. The EC consisted of five different door configurations while simulating a travel distance that an individual might experience in a relatively short emergency evacuation (Figure 1). The circuit was $48 \mathrm{~m}$ long and well-lit with a tile floor with the exception of one stretch that featured a carpeted gait mat, which allowed spatiotemporal gait measurements based on footfall locations and orientations. Each door featured a unique combination of opening direction, handle, latch, closer, and required opening force (Table 2). The opening force for each door was characterized using standard ADA protocol [21]. All doors were closed but unlocked and no other obstructions were present. The subjects were instructed to traverse the EC as quickly as possible without running, as if it were an emergency evacuation situation. The instruction to move "as quickly as possible without running" is typical in $6 \mathrm{MW}$ tests, but the addition of "as if it were an emergency situation" is unique to this study. Upon completion, the subject was provided a minimum of 10 min of seated rest.

In Phase 3, each subject completed a $6 \mathrm{MW}$ to simulate an extended walking distance. The $6 \mathrm{MW}$ used the EC path except that the segment to Door 5 was

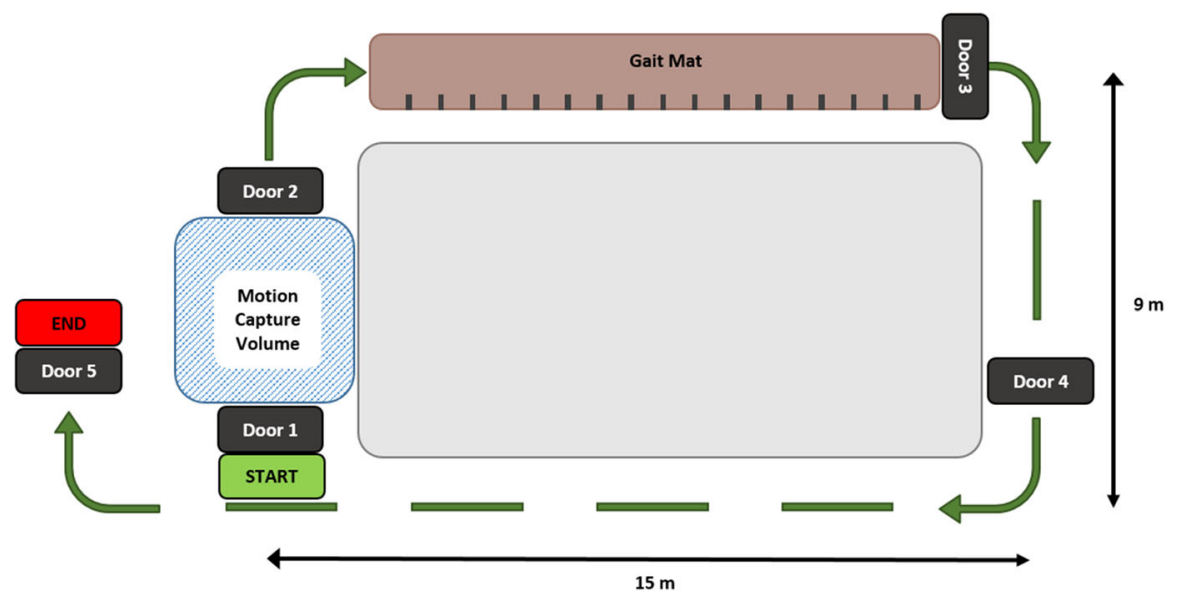

Figure 1. Evacuation circuit arrangement. Subjects began outside Door 1, moved through the motion capture space, through Door 2, along the carpeted gait mat, through Door 3 and Door 4, and completed the course after passing through Door 5 . 


\section{Table 2 \\ Each Door Presented a Unique Combination of Opening Direction, Hardware, Latch and Closer Mechaninsm and Required Opening Force}

\begin{tabular}{lclccc}
\hline Door & Direction & Hardware & Latch & Closer & Opening Force (N) \\
\hline Door 1 & Pull & Lever/twist & Yes & Yes & 33.7 \\
Door 2 & Push & Push plate & No & Yes & 30.8 \\
Door 3 & Pull & Lever/twist & Yes & No & 5.2 \\
Door 4 & Pull & Lever/twist & Yes & Yes & 50.4 \\
Door 5 & Push & Panic & Yes & Yes & 28.8 \\
\hline
\end{tabular}

excluded, all doors were open, and travel was in the opposite direction (counterclockwise). The $6 \mathrm{MW}$ is a reliable measure of physical capacity and fatigue during submaximal exercise $[22,23]$ and has been specifically validated for individuals with MS [3, 5, 24-26]. Subjects were instructed to walk as fast and as far as possible for six minutes. A researcher followed the subject with a measuring wheel (Stanley MW50, New Briton, CT) to record total distance travelled. Gait parameters were measured during the $6 \mathrm{MW}$ using a gait mat and motion capture system. Metabolic data were collected using a $\mathrm{K} 4 \mathrm{~b}^{2}$ portable monitoring unit (Cosmed S.r.l.; Rome, Italy). At th'e completion of the $6 \mathrm{MW}$, subjects stopped walking and were transported by wheelchair to the start of the EC.

For Phase 4, the subject then repeated the EC as soon as possible after completing the $6 \mathrm{MW}$ to characterize the potential impact of fatigue on traversing the doorways and egress after extended duration walking.

\subsection{Data Collection}

Total EC completion time and individual door passage times were measured during Phase 2 and Phase 4. Door passage times were recorded as the time between subject contact with the door or handle and contact of the trailing limb on the ground beyond the door threshold. EC completion time was split into door passage time (time spent passing through doors) and walking time (time travelling between doors). The sum of door passage times was subtracted from total time to determine walking time.

Gait parameters (described below) and the Oxygen Cost of Walking $\left(\mathrm{O}_{2} \mathrm{CW}\right)$ were computed from data collected in Phase 3. $\mathrm{O}_{2} \mathrm{CW}$ was computed as the steady state oxygen consumption $\left(\mathrm{VO}_{2}\right)$ divided by the walking velocity and averaged over the final three minutes of the $6 \mathrm{MW}$ (as in Sandroff [7]).

2.3.1. Spatiotemporal Gait Parameters To characterize spatiotemporal gait parameters, 23 reflective passive markers were applied to each subject (Figure 2). Five markers were placed on each foot: first toe, fifth toe, medial malleolus, lateral malleolus, and heel. Three markers were applied to each knee: lateral knee, medial knee, and tibia. One marker was applied to the lateral side of each thigh. Two markers were applied to each hip: anterior superior iliac spine and greater trochanter. One marker was applied to the sacrum. Marker locations were continu- 
ously recorded at $200 \mathrm{~Hz}$ as subjects walked between Doors 2 and 1 on each lap of the $6 \mathrm{MW}$ (Figure 1) using an eight camera motion capture system (OQUS 100, Qualisys AB; Sweden).

Three motion capture-based gait parameters were examined: average step width (avgSW), maximum step width (maxSW), and maximum step length (maxSL) (Figure 3). While several other common gait parameters can be quantified from the motion capture method, these measurements are reported here to characterize the local area that might be occupied during walking. Step width was defined as the distance in the y-direction (medial-lateral, perpendicular to the direction of travel) between the heels of the left and right feet. Step length was defined as the distance in the $\mathrm{x}$-direction (anterior-posterior, parallel to direction of travel) between the two heels. The trajectory of each heel was defined by assigning an index for the first and last frame in which the marker was captured by the camera. An interval was defined between the greater of the two indices (left or right) for initial appearance and the lesser of the two indices (left or right) for final appearance. Step length and step width were defined continuously on this interval. Maximum values for step length and step width were recorded, as well as an average for the step width across the entire interval. One subject from each population (MS, AMC, and YC) was excluded due to a lack of marker visibility.

Velocity was calculated by the gait mat software as the forward speed of the subject over the $7.9 \mathrm{~m}$ length of the gait mat (GAITRite, Platinum, CIR Systems; Sparta, NJ). Gait mat data over this open stretch of the EC allow characterization of uninterrupted gait speed, which is complimentary to the total distance travelled during the $6 \mathrm{MW}$ (that also includes the impact of accelerating and decelerating while turning corners, etc.). Measurement on each lap completed also provides the ability to quantify changes that may be attributed due to fatigue during the $6 \mathrm{MW}$.

\subsection{Data Analysis}

Data were analyzed using repeated measures ANOVAs (SPSS 23, IBM; Armonk, New York). For all measurements, comparisons were made between Groups (MS, AMC, YC). During the EC (i.e. Phases 2 vs 4), effects of Time were evaluated (pre- and post-6 MW), as well as comparisons between Doors (configuration of 5 specific doors). Both Time and Door main effects are included as repeated measures factors. Group, Time, and Door main effects and all interactions were examined where appropriate. Two-sided Pearson correlations for EC completion time and door passage time against EDSS score were completed. For the $6 \mathrm{MW}$, changes in velocity between the first and last lap (Fatigue main effect) were also studied. Significance was set at $p<0.05$.

\section{Results}

\subsection{Evacuation Circuit (EC)}

3.1.1. Time to Completion There was a significant Group effect on total completion time $(p<0.001)$. The MS population was significantly slower than either con- 


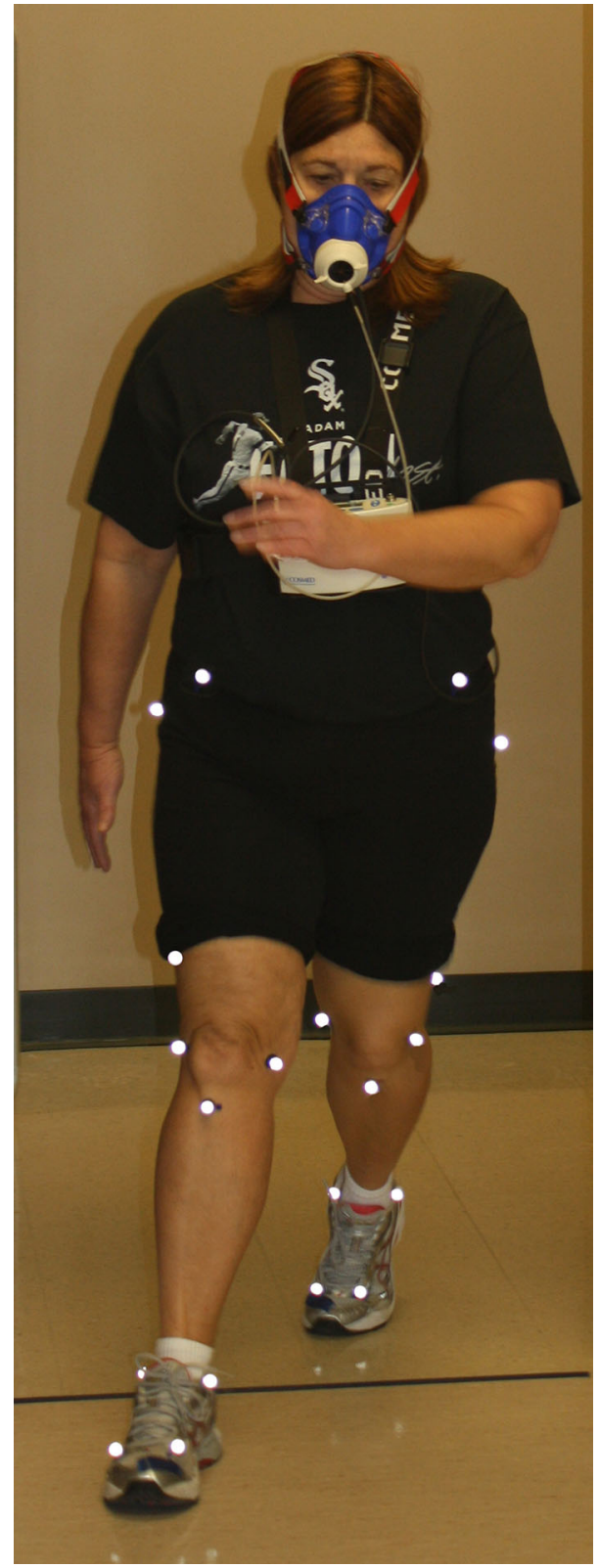

Figure 2. Twenty-three passive motion capture markers were placed on each subject.

trol group. There were no differences in evacuation time between the control groups. Further, there were no significant differences in EC completion time before and after the $6 \mathrm{MW}$ (no Time main effect) and no interaction effects. A 


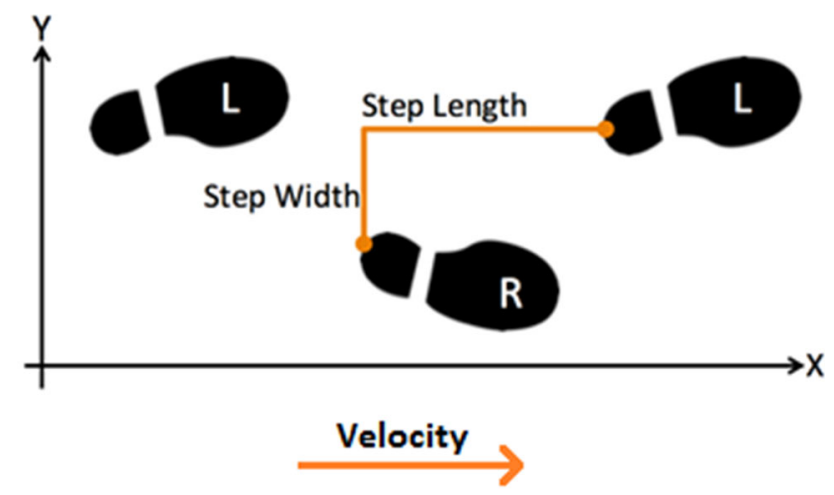

\section{Figure 3. Gait parameters examined included step width and step length.}

strong linear relation was observed between completion time and EDSS score ( $R=0.752, p=0.001)$, indicating higher EDSS scores resulted in longer times to complete the EC. Subjects took significantly longer walking between doors than passing through doors $(75.3 \%$ walking and $24.7 \%$ passing through doors, $p<0.001$, Figure 4). There were no differences in the percentage of time spent in door passage and walking before and after the $6 \mathrm{MW}$ or between the populations.

3.1.2. Door Passage All subjects were able to open all doors encountered. There was a significant Group effect (MS significantly slower than AMC and YC, $p<0.001$ ), but no significant effects of Time for door passage time (Figure 5a). Door design did result in significant differences in door passage time (Door main effect, $p<0.001$ ) (Figure 5b). Passage time for both push-direction doors (doors 2 and 5) was shorter than the passage time for all three pull-direction doors $(1,3$, and 4). Door 4 required the largest opening force and had the longest passage time. There was no statistical difference between doors 1 and 3. Further, there were no interaction effects. Average door passage time had a strong linear relation with EDSS score $(R=0.722, p=0.002)$, indicating higher EDSS scores resulted in longer door passage times.

\subsection{Six Minute Walk (6 MW)}

3.2.1. Gait Analysis A significant Group main effect was detected for avgSW $(p=0.002)$, maxSW $(p=0.017)$, maxSL $(p=0.002)$, and velocity $(p<0.001)$ (Table 3). The MS population had increased $\operatorname{avgSW}(p=0.001, p=0.005)$ and $\operatorname{maxSW}(p=0.015, p=0.011)$, decreased $\operatorname{maxSL}(p=0.006, p=0.01)$, and slower velocity $(p<0.001, p<0.001)$ relative to the control populations (AMC and $\mathrm{YC}$, respectively). There were no statistical differences between the age-matched controls and the young controls for any gait parameter. 


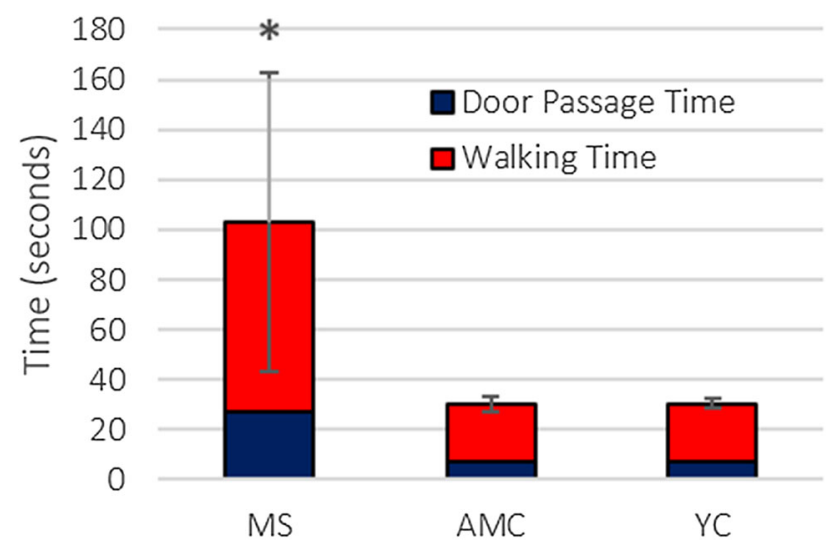

Figure 4. Averaged evacuation circuit completion time by population across both evacuation trials (pre- and post-6 MW). Completion time is split into door passage time and walking time. *MS was significantly different from all others $(p<0.05)$. Error bars indicate SE.
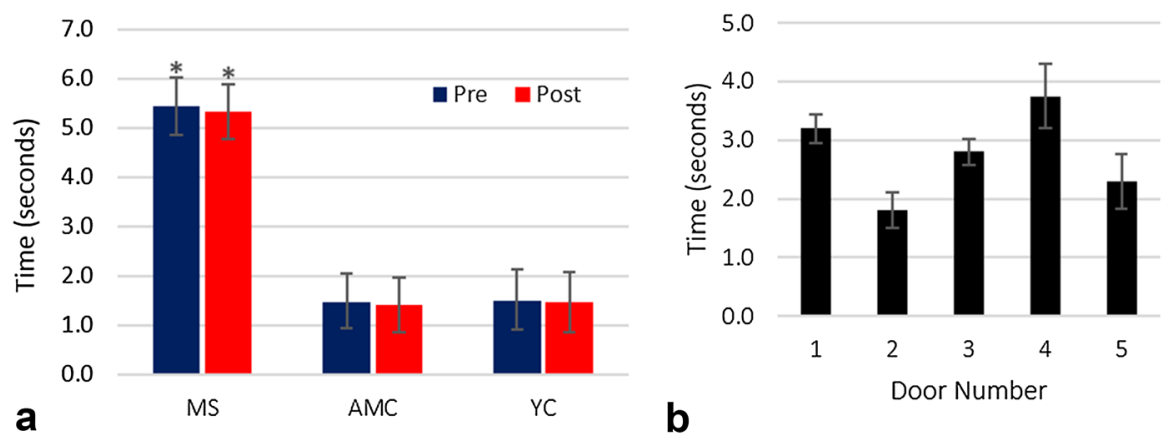

Figure 5. a Door passage time by Group and Time. *MS significantly different from all others ( $<<0.05)$. b Door passage time for all popuIations by door across both evacuation frials (pre- and post-6 MW). No statistical difference between 1 and 3 (NS); all others are statistically different from each other $(p<0.05)$. Error bars indicate SE.

There was a significant Fatigue main effect for straight-line gait velocity between the first and last laps of the $6 \mathrm{MW}$ for each group ( $p=0.005$, Table 4$)$ with the first lap faster than the last lap for each group. There were no statistically significant interaction effects for Group and Fatigue.

3.2.2. Distance Travelled There was a significant Group main effect for distance travelled in the $6 \mathrm{MW}(p<0.001)$. Distance travelled was significantly less for individuals with MS than AMC and YC $(p<0.001, p<0.001)$ (Figure 6). AMC and $\mathrm{YC}$ did not have a significant difference in travel distance. 


\section{Table 3}

Gait Parameters During Six Minute Walk by Group (mean \pm SE)

\begin{tabular}{lccc}
\hline Measure & \multicolumn{3}{c}{ Multiple sclerosis (MS) Age matched controls (AMC) Young controls (YC) } \\
\hline Average step width (mm) & $143 \pm 9^{*}$ & $100 \pm 9$ & $103 \pm 10$ \\
Maximum step width (mm) & $265 \pm 16^{*}$ & $210 \pm 15$ & $203 \pm 17$ \\
Maximum step length (mm) & $721 \pm 41^{*}$ & $887 \pm 40$ & $936 \pm 45$ \\
Average velocity (m/s) & $0.75 \pm 0.07^{*}$ & $1.98 \pm 0.07$ & $1.83 \pm 0.08$ \\
\hline
\end{tabular}

* MS significantly different from AMC and YC $(p<0.05)$

3.2.3. Metabolic Analysis The oxygen cost of walking $\left(\mathrm{O}_{2} \mathrm{CW}\right)$ for the $\mathrm{MS}$ population was significantly higher than all other populations indicating a significant Group main effect $(p=0.003)$ (Figure 7). Those with MS required more energy per meter of movement. There were no significant differences between the control groups.

\section{Discussion}

Individuals with MS required significantly longer times to traverse the evacuation circuit with various doorways, as was hypothesized. That is, those with MS took more than three times as long to complete the $48 \mathrm{~m}$ long EC relative to the control groups (102 versus $31 \mathrm{~s}$ ). The longer completion time in the MS group may be attributed to increased difficulty to open and pass through each door as well as changes in gait that resulted in decreased velocity of the MS group relative to the control groups (as measured during the $6 \mathrm{MW}$ ). These differences in walking patterns are commonly indicative of a more conservative gait, a compensation strategy that those with MS may use to maintain balance. Previous studies agree that individuals with MS walk with fewer, shorter, wider steps with a slower overall velocity $[4,6]$. The increased step width suggests that individuals with MS may occupy a larger base of support and require more space to walk naturally and comfortably during an evacuation. Slower velocity in the MS group may be explained by decreased aerobic capacity [3, 6], a finding also reflected in the increased $\mathrm{O}_{2} \mathrm{CW}$ reported in the MS population in this study.

\section{Table 4}

\section{Speed Differences Between First and Last Laps During 6 MW} ( $m e a n \pm$ SE)

\begin{tabular}{lccc}
\hline Measure & $\begin{array}{c}\text { Multiple } \\
\text { sclerosis (MS) }\end{array}$ & $\begin{array}{c}\text { Age matched } \\
\text { controls (AMC) }\end{array}$ & $\begin{array}{c}\text { Young } \\
\text { controls (YC) }\end{array}$ \\
\hline Velocity during First Lap (m/s) & $0.81 \pm 0.40$ & $2.05 \pm 0.24$ & $1.87 \pm 0.21$ \\
Velocity during Last Lap (m/s) & $0.69 \pm 0.34 *$ & $1.90 \pm 0.21 *$ & $1.81 \pm 0.29 *$ \\
\hline
\end{tabular}

* Last Lap significantly different from First Lap $(p<0.05)$ 


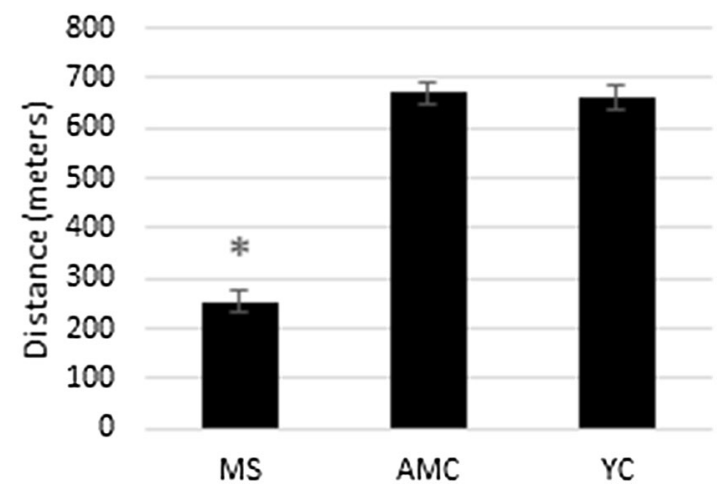

Figure 6. $6 \mathrm{MW}$ Distance travelled by population during $6 \mathrm{MW}$. *MS was significantly different from all others $(p<0.05)$. Error bars indicate SE.

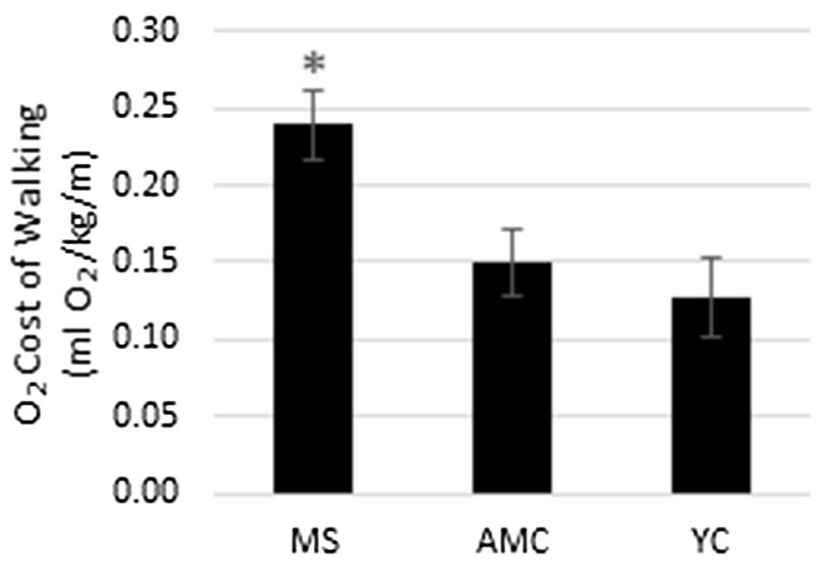

Figure 7. Oxygen cost of walking by population during 6 MW. *MS significantly different from all others $(p<0.05)$. Error bars indicate SE.

For all three groups, there was a significant decrease in velocity between the first and last lap on the gait mat during the $6 \mathrm{MW}$ (Table 4). This decrease in velocity, possibly due to induced fatigue, was hypothesized; however, there were no differences in the EC completion time (Phases 2 and 4) before and after the 6 MW. Previous studies have shown that MS populations slow over the duration of the $6 \mathrm{MW}$, while healthy control groups initially slow, but return to the initial speed or greater during the final minute of the $6 \mathrm{MW}$ [23]. Perhaps there was a response within the MS population that enabled them to match initial EC completion time despite being fatigued from the $6 \mathrm{MW}$. During the $6 \mathrm{MW}$, the MS population covered a smaller distance (slower average velocity) with slower straight-line velocities. Interestingly, the average velocity across the entire $6 \mathrm{MW}$ was lower than the straight-line velocities in the MS $(0.70$ vs $0.75 \mathrm{~m} / \mathrm{s})$ and AMC $(1.86$ vs. $1.98 \mathrm{~m} / \mathrm{s})$ 
groups, but was not different in the YC group (1.83 vs. $1.83 \mathrm{~m} / \mathrm{s})$. While turning corners and the passage through the doorways, even with the doors fixed open, resulted in decreased velocity in the older groups, the younger subjects traversed these obstacles without apparent modification to their overall speed. Importantly, the overall slower pace in the MS population can locally reduce flow of evacuees, increasing overall egress time even for those without movement disorders.

Additionally in our study, there was variability within individuals with MS, related to the severity of disease progression. EDSS is scored based on ambulatory ability, with a higher EDSS score indicating decreased mobility. High EDSS scores have been directly related to decreased $6 \mathrm{MW}$ distance travelled [3, 23], and is positively correlated with time to complete the evacuation circuit and door passage time in the current study $(R=0.752$ and $R=0.722$, respectively). These results suggest that evacuation in an emergency scenario would take significantly longer for individuals with more severe MS who may have decreased egress capabilities. The age-matched and young controls experienced similar EC completion times, indicating that age was not a major factor for evacuation time in this study. This result is contrary to previous research indicating decreasing $6 \mathrm{MW}$ distance with increased age, though age ranges in that study were from 60 to 89 [27]. The age-matched control population in the current study averaged about 50 years, perhaps young enough to avoid the effects observed by Steffen et al.

The results from door passage time suggest that door passage is fastest when the door does not involve a latch or a handle and that traversing a push-direction door is quicker than traversing a pull-direction door. Typically, doors in common area egress pathways are designed to open in the direction of travel for safer evacuation, and the results of this study further enforce this standard. The passage times in this study are slightly faster than those reported by Boyce [13], perhaps because the subjects in the present study were told to navigate the doors as they would in an emergency. Boyce [13] reported longer passage times and increased failure rates with doors requiring greater opening force. Interestingly, in this study, there was no statistical difference between the door passage time for Doors 1 and 3, with opening forces of $33.7 \mathrm{~N}$ and $5.2 \mathrm{~N}$, but these were significantly shorter than Door 4 that required $50.4 \mathrm{~N}$ of force. This study suggests that the door passage time for the MS population is more heavily influenced by direction than opening force. Additionally, while there is a trend for increasing passage time for pull doors with increasing opening force, passage time was only significantly different for the heaviest door. This finding may suggest a potential threshold value under which the ability to open and traverse the door is not affected by required opening force. Finally, while Boyce [13] reported some failures to operate doors at forces as low as $21 \mathrm{~N}$, our population was able to open and traverse all door conditions presented.

While this study provides valuable new feasibility, pilot data on egress in MS, the sample size was relatively small. Future researchers should recruit larger and more heterogeneous samples of MS, for further understanding our novel results. The results of this study could be further expanded through a larger motion capture volume with an increased subject base to better characterize the spectrum of gait deficiencies related to MS and other movement disorders. The study popula- 
tion could also be expanded to include those who require the use of a wheelchair or other assist device and to include young adults with MS.

\section{Conclusion}

The portion of the population with movement disorders is projected to increase in future decades. As these populations become more prevalent in society, considerations must be made for individuals with movement disorders in regard to evacuation planning. This study illustrates the increased evacuation time and door passage time of individuals with MS, possibly as a result of altered gait parameters. Further, these changes become more pronounced as disease progression increases. Individuals with MS display wider, shorter steps as well as slower velocity than age-matched or young controls. Improved understanding of the spatial aspects of gait in those with MS can help in the design of evacuation simulations and building development. In this study, individuals with MS required more physical space, particularly width, during gait, which may play an important role in evacuation scenarios, when considering crowd density or the maximum capacity of an egress pathway. Overall evacuation of a structure is depended on many factors including population density and the actions of others, but improved movement data for individuals, especially those with movement disorders, is essential when considering evacuation scenarios. These data are particularly important for buildings located in areas with high populations of aging or disabled individuals, especially as these individuals gain improved access. This study can serve as an important step towards obtaining detailed evacuation characteristics for individuals with movement disorders.

\section{Acknowledgements}

Funding support for MB was provided by the National Science Foundation Engineering Research Center for Compact and Efficient Fluid Power (0540834), with additional support from the Foundation of the Consortium of Multiple Sclerosis Centers' Multiple Sclerosis Workforce of the Future program.

\section{Compliance with Ethical Standards}

Conflict of inferest There are no conflicts of interest regarding this work.

\section{Open Access}

This article is distributed under the terms of the Creative Commons Attribution 4.0 International License (http://creativecommons.org/licenses/by/4.0/), which permits unrestricted use, distribution, and reproduction in any medium, provided you give appropriate credit to the original author(s) and the source, provide a link to the Creative Commons license, and indicate if changes were made. 


\section{References}

1. Bach J-P, Ziegler U, Deuschl G, Dodel R, Doblhammer-Reiter G (2011) Projected numbers of people with movement disorders in the years 2030 and 2050. Mov Disord 26(12):2286-2290. doi:10.1002/mds.23878

2. (2005) Multiple sclerosis information sourcebook, N.M.S. Society, Editor., Information Resource Center and Library of the National Multiple Sclerosis Society, New York

3. Chetta A, Rampello A, Marangio E, Merlini S, Dazzi F, Aiello M, Ferraro F, Foresi A, Franceschini M, Olivieri D (2004) Cardiorespiratory response to walk in multiple sclerosis patients. Respir Med 98(6):522-529

4. Sosnoff JJ, Sandroff BM, Motl RW (2012) Quantifying gait abnormalities in persons with multiple sclerosis with minimal disability. Gait Posture 36(1):154-156. doi:10.1016/ j.gaitpost.2011.11.027

5. Pilutti LA, Dlugonski D, Sandroff BM, Suh Y, Pula JH, Sosnoff JJ, Motl RW (2013) Gait and six-minute walk performance in persons with multiple sclerosis. J Neurol Sci 334(1-2):72-76. doi:10.1016/j.jns.2013.07.2511

6. Sandroff BM, Sosnoff JJ, Motl RW (2013) Physical fitness, walking performance, and gait in multiple sclerosis. J Neurol Sci 328(1-2):70-76. doi:10.1016/j.jns.2013.02.021

7. Sandroff BM, Klaren RE, Pilutti LA, Motl RW (2014) Oxygen cost of walking in persons with multiple sclerosis: Disability matters, but why? Multiple Sclerosis International 2014:7. doi:10.1155/2014/162765

8. Novotna K, Sobisek L, Horakova D, Havrdova E, Lizrova Preiningerova J (2016) Quantification of gait abnormalities in healthy-looking multiple sclerosis patients (with expanded disability status scale 0-1.5). Eur Neurol 76(3-4):99-104. doi:10.1159/ 000448091

9. Motl RW, Learmonth YC (2014) Neurological disability and its association with walking impairment in multiple sclerosis: brief review. Neurodegener Dis Manag 4(6):491500. doi: $10.2217 / \mathrm{nmt} .14 .32$

10. Boyce KE, Shields TJ, Silcock GWH (1999) Toward the characterization of building occupancies for fire safety engineering: prevalence, type, and mobility of disabled people. Fire Technol 35(1):35-50. doi:10.1023/a:1015335132296

11. Boyce KE, Shields TJ, Silcock GWH (1999) Toward the characterization of building occupancies for fire safety engineering: capabilities of disabled people moving horizontally and on an incline. Fire Technol 35(1):51-67. doi:10.1023/A:1015339216366

12. Boyce KE, Shields TJ, Silcock GWH (1999) Toward the characterization of building occupancies for fire safety engineering: capability of people with disabilities to read and locate exit signs. Fire Technol 35(1):79-86. doi:10.1023/a:1015395318183

13. Boyce KE, Shields TJ, Silcock GWH (1999) Toward the characterization of building occupancies for fire safety engineering: capability of disabled people to negotiate doors. Fire Technol 35(1):68-78. doi:10.1023/a:1015391217275

14. Shields TJ, Smyth B, Boyce KE, Silcock GWH (1999) Evacuation behaviours of occupants with learning difficulties in residential homes. Disabil Rehabil 21(1):39-48. doi:10.1080/096382899298089

15. Peacock RD, Hoskins BL, Kuligowski ED (2011) Overall and local movement speeds during fire drill evacuations in buildings up to 31 stories. In: Peacock RD, Kuligowski ED, Averill JD (eds) Pedestrian and evacuation dynamics Springer, Boston, pp 25-35

16. Kuligowski ED, Hoskins BL (2010) Occupant behavior in a high-rise office building fire. NIST Technical Note 1664. National Institute of Standards and Technology

17. Kuligowski E, Peacock R, Wiess E, Hoskins B (2015) Stair evacuation of people with mobility impairments. Fire Mater 39(4):371-384. doi:10.1002/fam.2247 
18. Jiang CS, Zheng SZ, Yuan F, Jia HJ, Zhan ZN, Wang JJ (2012) Experimental assessment on the moving capabilities of mobility-impaired disabled. Saf Sci 50(4):974-985. doi:10.1016/j.ssci.2011.12.023

19. Golmohammadi D, Shimshak D (2011) Estimation of the evacuation time in an emergency situation in hospitals. Comput Ind Eng 61(4):1256-1267. doi:10.1016/ j.cie.2011.07.018

20. Kurtzke JF (1983) Rating neurologic impairment in multiple sclerosis: an expanded disability status scale (EDSS). Neurology 33(11):1444-1452

21. Justice DO (2010) ADA standards for accessible design. In: Chapter 4: Accessible Routes

22. Butland RJ, Pang J, Gross ER, Woodcock AA, Geddes DM (1982) Two-, six-, and 12minute walking tests in respiratory disease. Br Med J (Clin Res Ed) 284(6329):16071608

23. Goldman MD, Marrie RA, Cohen JA (2008) Evaluation of the six-minute walk in multiple sclerosis subjects and healthy controls. Mult Scler 14(3):383-390. doi:10.1177/ 1352458507082607

24. Kervio G, Carre F, Ville NS (2003) Reliability and intensity of the six-minute walk test in healthy elderly subjects. Med Sci Sports Exerc 35(1):169-174. doi:10.1097/00005768200301000-00025

25. Savci S, Inal-Ince D, Arikan H, Guclu-Gunduz A, Cetisli-Korkmaz N, Armutlu K, Karabudak R (2005) Six-minute walk distance as a measure of functional exercise capacity in multiple sclerosis. Disabil Rehabil 27(22):1365-1371

26. Motl RW (2013) Ambulation and Multiple Sclerosis. Physical Medicine and Rehabilitation Clinics of North America 24(2):325-336. doi:10.1016/j.pmr.2012.11.004

27. Steffen TM, Hacker TA, Mollinger L (2002) Age- and gender-related test performance in community-dwelling elderly people: six-Minute Walk Test, Berg Balance Scale, Timed Up \& Go Test, and gait speeds. Phys Ther 82(2):128-137 